\title{
Different Approaches in Uncertainty Evaluation for Measurement of Complex Surfaces Using Coordinate Measuring Machine
}

\author{
Igor $\mathrm{Vrba}^{1}$, Rudolf Palencar ${ }^{1}$, Miodrag Hadzistevic ${ }^{2}$, Branko Strbac ${ }^{2}$, Vesna Spasic-Jokic ${ }^{2}$, \\ Janko Hodolic ${ }^{2}$ \\ ${ }^{1}$ Faculty of Mechanical Engineering, Institute of Automation, Measurement and Applied Informatics, Slovak Technical \\ University, 81243 Bratislava, Nám. slobody 17, Slovak Republic, e-mail: igor.vrba@stuba.sk, rudolf.palencar@stuba.sk \\ ${ }^{2}$ Faculty of Technical Sciences, University of Novi Sad, 21000 Novi Sad, Trg Dositeja Obradovica 6, Serbia \\ e-mail: svesna@uns.ac.rs,miodrags@uns.ac.rs, strbacb@uns.ac.rs, hodolic@uns.ac.rs
}

\begin{abstract}
This paper describes a methodology for uncertainty assessment for Coordinate Measuring Machine measurement of complex real work pieces from industry. The study applied two approaches (in scanning mode only) for estimating the measurement uncertainty with the support of Taguchi plan in the experiment containing five factors: scanning speed, sample density, probe configuration, scanning direction, and position of measuring object. In the first approach the uncertainty was estimated by measuring the basic geometric objects (primitives like sphere and torus) representing the decomposition of complex surfaces and in the second one a complex surface was treated as an unknown quantity. Calculated uncertainty Type A for both measurement tasks was in the range from $0.65 \mu \mathrm{m}$ to $6.47 \mu \mathrm{m}$. Evaluation of the uncertainty Type B covered specifications of the machine and standard uncertainties derived from temperature effects. Total $u_{B}$ component was found to be in order of $0.4 \mu \mathrm{m}$. Future research will be directed towards the development and application of simulation methods
\end{abstract}

Keywords: Coordinate measuring machine, uncertainty, complex surfaces, traceability.

\section{INTRODUCTION}

$\mathrm{M}$ EASUREMENT UNCERTAINTY is a parameter associated with measurement result and presents an assessment that is characterized by a range of values within which the measured value is. Complete measurement reports must contain statement about the measurement uncertainty. This fact is relevant due to two important metrological concepts, i.e. decision on "the acceptance or rejection" and maintaining traceability in accordance with ISO 9000 standards [1], [2]. Lately, coordinate measuring machines (CMM) have become dominant in production metrology. They are also successfully applied for the inspection of complex surfaces, i.e. multiplex surfaces (freeform surfaces) which belong to the most complex measuring tasks.

Complex workpieces are increasingly common in industrial practice and recently a lot of work has been done in the development of methods and techniques for their measuring on CMM [3]. The results obtained by CMM are often the basis for making decisions with significant economic consequences. Therefore, the determination of the measurement uncertainty at CMM has become one of the most recent areas of research in the last two decades. There are many factors that influence the measurement uncertainty. They have also strong mutual influence, so that the measurement uncertainty is strongly dependent on measuring specificities tasks.

Several methods for determining the measurement uncertainty of a specific measurement task were described in the review paper [4]. Some of these methods are documented in the ISO 15530 series of standards: the use of multiple strategy measurements without calibrated workpiece 15530-2 (deleted), the use of calibrated workpieces or standards 15530-3 [5], and the use of computer simulation 15530-4 [6]. Uncertainty estimation is further complicated when measuring compound surfaces (complex surfaces) at CMM, which represents a special direction of research. Complex parts significantly complicate the measuring process at CMM, as the existing standards and recommendations for inspection at CMM cannot be fully applied and some new errors and stochastic uncertainties occur. The main problem in uncertainty assessment of the CMM in measuring complex shapes is to ensure the traceability of measurements, i.e. it is difficult to calibrate really complex surfaces with high precision.

Savio and De Chiffre have presented work detailing the validation of calibration procedures for free-form parts on CMMs [7]. This paper is relevant for our work and describes a new method for the establishment of traceability of freeform measurements made on CMMs. They have also presented the concept of "calibrated workpieces" and additionally developed Modular Freeform Gauge (MFG) [8]. In this concept the freeform surface is substituted by the surfaces of simple objects, assembled in such a way that the shape of interest is simulated as closely as possible. The MFG concept has practical limitations with respect to feasible configurations and similarity requirements; however, it can help in establishing traceability from actual freeform object measured on the CMM through MFG, regular object with regular geometry and length-measuring capability of CMMs to SI unit of meter. In this case uncertainty was estimated according to ISO/TS 15530-3. Uncertainty budget consisted of several factors: the uncertainty of the calibration of individual objects, uncertainty of relative positions in MFG configuration, the 
uncertainty of the measurement procedure (repeated measurements), the uncertainty due to variations of measuring objects, and uncertainty due to the reduction in the similarity level between MFG and real objects. The major limitation of the procedure called "Using a calibrated workpiece" lies in the artifact. In order to overcome this lack, Savio et al. have proposed a method of "Using uncalibrated workpieces" for uncertainty estimation for measuring the complex shapes using CMMs [9]. Basically, the experiment includes variation of uncertainty factors and measurements were performed repeatedly. In this way it is possible to determine the reproducibility of measurements, but this procedure does not take into account the systematic component of error and the duration of experiment is too long.

Barini et al. presented experimental uncertainty assessment for complex surfaces using available artifacts and varying the parameters of the measuring process, which is, generally, a combination of the previous two methods [10]. The total uncertainty consisted of four standard uncertainties due to: calibration of the objects which formed a complex surface; calibration of the relative position of objects; measurement procedure and the thermal effects. From the uncertainty budget it is obvious that the greatest impact on the overall uncertainty has the uncertainty of measurement procedures. For the first time the concept of Design of Experiment (DOE) was applied to explore the effects of different measurement process parameters on the measurement uncertainty in the case of complex surfaces. This methodology should be extended to those parts of industrial production which do not have calibrated artifacts.

An analysis of previous investigations leads to some conclusions as are: for uncertainty assessment and traceability maintenance of complex surface measured by CMM it is necessary to use a calibrated artifact that simulates a complex surface; measurement uncertainty can be determined without calibrated artifacts using the varying of the measuring process parameters, but in this case traceability was not assured; examining the budget of uncertainty [10] when the calibrated artifact is applied and measurement process parameters are varied using DOE concept; it was found that the highest uncertainty arises from the measurement procedure.

The review of literature also clarifies that: there is no specific ISO standard that addresses current free-form requirements; there are no physical standards currently available to support an industry that is becoming more reliant on free-form measuring systems; more and more sophisticated free-form measuring systems are being designed and are entering the market place; verified freeform metrology capabilities are essential for industry, biomedical research, etc.; development of a free-form verification process and supporting artifacts is demanded by industry and health services. Also, no precise definition for a free-form surface was found. When trying to describe a freeform surface, even the use of 'asymmetry' can be misleading, as it may be argued that if one axis of a surface is described mathematically as a geometric shape, it cannot form part of a truly free-form surface. However, there are countless numbers of components and artifacts that combine a series of varying shapes and geometries. Whilst discrete regions of an artifact such as this may be described mathematically, the whole artifact has a complex form and is therefore very difficult to describe by a simple mathematical expression.

The ISO 10360 series of standards detail the acceptance, reverification tests and interim checks required to determine whether the CMM performs to the manufacturer's stated maximum permissible error of length measurement. The user should develop task-related measuring strategies for each measurement undertaken that will provide the appropriate level of confidence in the overall result [11].

Methodology for uncertainty assessment with aim to assure traceability of CMM measurement is of crucial importance. Mutual recognition arrangement in metrology requires complete traceability from the measurement in the user's laboratory through accredited laboratories to national primary standards and further to the definition and realization of measuring unit. From the stand point of legal metrology it is easy to achieve through regional and international key and supplementary intercomparisons [12], [13].

Feautrier and Bourdet [15] describe a method for separating the errors in a free-form component from the errors present in the CMM performing the measurements. The method builds on the various repositioning and reversal techniques used in dimensional metrology. The technique would be useful to pursue where traceability is required or when form errors need to be measured with uncertainties of a few micrometers, but it has less relevance in the cases where instrument uncertainties are tens of micrometers, but the manufactured free-form artifacts are likely to be calibrated to within $\pm 10 \mu \mathrm{m}$. For the case of measurement of 'standard' shapes, it may be possible to use the CMM as a comparator (against such artefacts as plain setting ring gauges and gauge blocks), such that the results can be said to be traceable [5].

Unlike previous studies, this paper gives a methodology for uncertainty assessment of CMM measurement of complex surfaces of real workpieces from industry. The study applied two approaches for estimating the measurement uncertainty with the support of Taguchi plan in the experiment. In the first approach the measurement uncertainty was estimated by measuring the basic geometric objects (primitives) representing the decomposition of complex surfaces and in the second approach a complex surface was treated as an unknown quantity (digitizing). True traceability in CMM measurements is difficult to achieve. The structure of the CMM is so complex that the error propagation is too difficult to analyze with sufficient accuracy to provide a reliable enough error budget to fulfill the strict traceability requirements. Only in a very restricted set of circumstances, primarily when the CMM is being used as a simple comparator, can the results be said to be traceable, ISO 10360 is generally accepted as a way of demonstrating traceability. 


\section{SUBJECT \& METHODS}

\section{A. Workpiece}

Work item which is exemplified by the complex surface is presented in Fig.1. It is designed for injection of plastic wrap of chocolate egg. CAD / CAM techniques were used in design and manufacture. Processing is performed on a multiaxial processing center and the high quality of surface was achieved by lapping. The whole unit represents a complex surface that can be subdivided into geometric features. When the complex surface is decomposed it is possible to obtain segments of the sphere and the torus (Fig.1.).

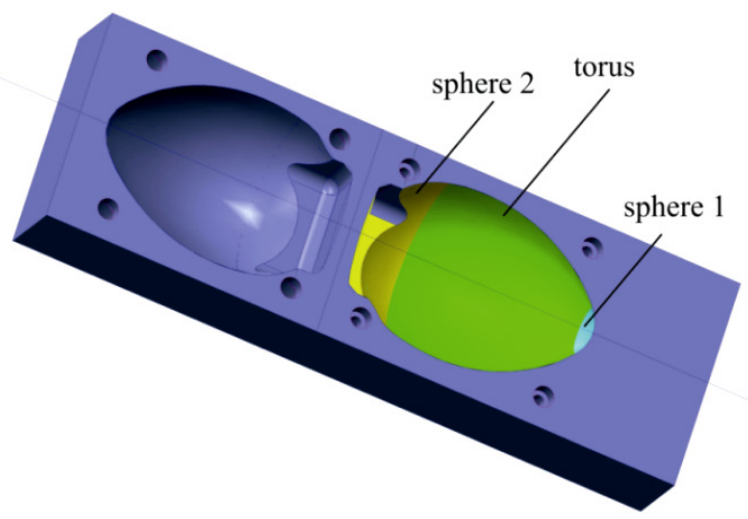

Fig.1. Complex workpiece surface separated into geometric features.

Basically, we have developed two measurement tasks. The first one was directed at the measurement of the workpiece as a set of geometric figures and the other one measures the workpiece as the unknown complex surface. Both measurement tasks were performed in the scanning mode, the most suitable for the acquisition of the number of points required for a reliable estimation. We used tactile system coordinate measuring machine. Typical CMM operates using the same principle, by moving a spherical ended stylus to make contact with the object to be measured at appropriate points.

\section{B. Uncertainty sources in CMM measurement}

Sources of errors in CMM measurements can be classified as spatial errors or computational errors. Spatial errors are errors in the measured position of a point on the surface of the workpiece and are determined by: the accuracy of the components of the CMM - the guideways, the scales, the probe system and the qualification sphere; the environment in which the CMM operates - the ambient temperature, temperature gradients, humidity and vibration; the probing strategy used - the magnitude and direction of the probe force, the type of probe stylus used and the measuring speed of the probe; and the characteristics of the workpiece elasticity, surface roughness, hardness and the mass of the component [11]. Computational errors are the errors in the estimated dimensions and form deviations of the workpiece and are determined by: the CMM software used to estimate the geometry of the workpiece; the precision of the computer used on the CMM; the number and relative position of the measured points; and the extent to which the geometry departs from the ideal geometric form [11]. Reference [14] shows typical CMM geometric accuracies which can be used in total uncertainty assessment.

Design of Experiments (DOE) is a group of techniques used to explore the relationship between several variables involved in a given phenomenon. It is a suitable technique for the detection of reproducibility of measurements under different measurement conditions and its applicability is closely related to ISO / TS 15530-2. From reproducibility of measurements it is possible to calculate standard deviation, which is the basis for estimating Type A uncertainty of measurement. There are several studies that have used experimental design for estimating uncertainty of CMM measurements. Approach for determining the measurement uncertainty of CMM through test performances consists of measuring calibrated ball-bars of different lengths in different orientations in the work space according to selected plan of the experiment [16]. Feng and associates also used DOE to investigate the effects of factors and their interactions on the CMM measurement uncertainty as it was defined in the GUM [17], [18]. This experiment confirmed that the uncertainty was minimized when the speed was the highest, probe the shortest, the ratio of the measuring sensor was the largest, and the number of points of the slope was the greatest. Sun et al. have studied the development of a comprehensive framework for the application of design of experiment in determining the measurement uncertainty of the CMM [19].

In this paper, instead of the factorial design we used Taguchi experiment modified plan to reduce the number of runs needed to explore the impact of variables. The plan of the experiment according to Taguchi contained five factors, each at two levels. The following factors were considered: scanning speed, sample density, probe configuration, scanning direction, and the position of the measuring objects on the CMM table, given in Table 1.

Table 1. Factors and levels of experimental plan.

\begin{tabular}{|c|c|c|c|}
\hline Factor & +Level & -Level & Code \\
\hline Scanning speed & 10 & 40 & $\mathrm{~A}$ \\
\hline Sample density & 2.5 & 0.65 & $\mathrm{~B}$ \\
\hline Probe configuration & $1.5 / 70$ & $3 / 33$ & $\mathrm{C}$ \\
\hline Scanning direction & $\mathrm{X}$ & $\mathrm{Y}$ & $\mathrm{D}$ \\
\hline Position on CMM & $\min (\mathrm{x}, \mathrm{y})$ & $\max (\mathrm{x}, \mathrm{y})$ & $\mathrm{E}$ \\
\hline
\end{tabular}

In addition to these factors the impact of the following interaction was investigated: (i) scanning speed and sample density $(\mathrm{A} \times \mathrm{B})$; (ii) scanning speed and probe configuration $(\mathrm{A} \times \mathrm{C})$; (iii) sample density and probe configuration $(\mathrm{B} \times \mathrm{C})$; (iv) scanning direction and position on $\mathrm{CMM}(\mathrm{D} \times \mathrm{E})$

The choice of factors was carried out according to the basic requirements that were placed in programming CMM for execution of measurement tasks in scan mode. Scanning speed and sample density represent the factors that cause uncertainty related to the measurement strategy but also for the object to be measured. In order to obtain more accurate 
measurements, i.e. the set of points that represents measured surface in the best way, it is preferable to keep the scanning speed at a minimum level and sample density at the maximum. But this practice is not economically justified.

The configuration of the measuring probe contributes to total uncertainty. The ratio $1.5 / 70$ represents the ratio of diameter of the top of the probe and the length of tentacle.

Scanning direction enters a component of uncertainty related to different behaviors and performance of measurement system between the $\mathrm{X}$ direction and $\mathrm{Y}$ direction. The position of the workpiece on the CMM table enters a component of uncertainty related to CMM hardware. If the factor is located on the first level then the workpiece is positioned close to the reference coordinate system of the machine, while the second factor level means that the workpiece is on the maximum transverse distance from the reference coordinate system of the machine. In order to ensure the reproducibility of the position of the workpiece at the table the measuring machines are fitted with two accessories.

According to Taguchi experimental design the applied matrix was L16 and it is given in Table 2. [20]. In the experimental design, five observations were carried out, a total of 80 fully randomized experiments in strict microclimate controlled laboratory was performed. We used a coordinate measuring machine, Carl Zeiss Contura G2 RDS equipped with contact VAST XXT scanning probe. Maximal permissible error was $\left(\mathrm{MPE}_{\mathrm{E}}\right) 1.9+L / 330 \mu \mathrm{m}(L$ is length expressed in $\mathrm{mm}$ ).

Table 2. Orthogonal array L16 of Taguchi.

\begin{tabular}{|c|c|c|c|c|c|c|c|c|c|}
\hline & \multicolumn{10}{|c|}{ Columns no. } \\
\hline $\begin{array}{c}\text { Test } \\
\text { No. }\end{array}$ & $\mathbf{A}$ & $\mathbf{B}$ & $\mathbf{A} \times \mathbf{B}$ & $\mathbf{C}$ & $\mathbf{A} \times \mathbf{C}$ & $\mathbf{B} \times \mathbf{C}$ & $\mathbf{D}$ & $\mathbf{E}$ & $\mathbf{D} \times \mathbf{E}$ \\
\hline 1 & 1 & 1 & 1 & 1 & 1 & 1 & 1 & 1 & 1 \\
\hline 2 & 1 & 1 & 1 & 1 & 1 & 1 & 1 & 2 & 2 \\
\hline 3 & 1 & 1 & 1 & 2 & 2 & 2 & 2 & 1 & 2 \\
\hline 4 & 1 & 1 & 1 & 2 & 2 & 2 & 2 & 2 & 1 \\
\hline 5 & 1 & 2 & 2 & 1 & 1 & 2 & 2 & 1 & 2 \\
\hline 6 & 1 & 2 & 2 & 1 & 1 & 2 & 2 & 2 & 1 \\
\hline 7 & 1 & 2 & 2 & 2 & 2 & 1 & 1 & 1 & 1 \\
\hline 8 & 1 & 2 & 2 & 2 & 2 & 1 & 1 & 2 & 2 \\
\hline 9 & 2 & 1 & 2 & 1 & 2 & 1 & 2 & 1 & 2 \\
\hline 10 & 2 & 1 & 2 & 1 & 2 & 1 & 2 & 2 & 1 \\
\hline 11 & 2 & 1 & 2 & 2 & 1 & 2 & 1 & 1 & 1 \\
\hline 12 & 2 & 1 & 2 & 2 & 1 & 2 & 1 & 2 & 2 \\
\hline 13 & 2 & 2 & 1 & 1 & 2 & 2 & 1 & 1 & 1 \\
\hline 14 & 2 & 2 & 1 & 1 & 2 & 2 & 1 & 2 & 2 \\
\hline 15 & 2 & 2 & 1 & 2 & 1 & 1 & 2 & 1 & 2 \\
\hline 16 & 2 & 2 & 1 & 2 & 1 & 1 & 2 & 2 & 1 \\
\hline
\end{tabular}

\section{Uncertainty evaluation}

Uncertainty assessments in both measurement tasks were performed according to the instructions contained in the Guide to the expression of uncertainty in measurement (GUM) [19], combining Type A and Type B uncertainties. In this paper the authors have focused on the assessment of the uncertainty that comes from the measurement process parameters, while other factors of uncertainty are not taken into account. This points to the incompleteness of measurement uncertainty budget, but it is important to establish a methodology.

Evaluation of the uncertainty in the measurement of complex surfaces such as decomposition of basic geometric primitives is based upon the well-known formula for expanded measurement uncertainty given by (1) [21].

$$
U_{\text {form }}=k \sqrt{u_{A}^{2}+u_{B}^{2}} \text {. }
$$

where $k$ is a coverage factor calculated according to values of degrees of freedom and the adopted level of confidence; $u_{\mathrm{A}}$ - uncertainty of Type A (reproducibility of measurements) obtained over five repetitions of the same experiment; $u_{\mathrm{B}}-$ uncertainty of Type $\mathrm{B}$ obtained by calculation from Maximum Permissible Error divided by coefficient of the implemented function of density distribution (MPE/ $\lambda$, in this particular case for uniform distribution $\lambda=\sqrt{3}$ ). The standard measurement uncertainty of Type A is calculated for all three geometric primitives and in all 16 experimental treatments was the highest for the case of torus. For $n$ repeated measurements, Type A uncertainty given by (3) is derived from independent statistical observations $x_{i, k}$ and $x_{i}$ under repeatable conditions and mean value (2): [21]

$$
\bar{x}=\sum_{i=1}^{n} x_{i} / n
$$

$$
u(x)=\sqrt{\frac{1}{n(n-1)} \sum_{i=1}^{n}\left(x_{i}-\bar{x}\right)^{2}}
$$

Evaluation of the uncertainty in the measurement of complex surfaces as an unknown area does not include uncertainty of Type B because in this case the reference size $L$, relevant for maximum permissible error (MPE) was not available. Therefore, in this case, the expanded uncertainty is calculated only over a factor of Type A uncertainty, given by (4):

$$
U_{\text {FreeForm }}=k u_{A}
$$

\section{Results}

The measurement result represented the deviation of complex surface shape from the ideal CAD model as the maximum and minimum deviation calculated along a nominal orthogonal vector. In the first mode of measurement, when the workpiece was decomposed into basic geometrical primitives, a deviation from the ideal shape sphere and torus has been followed. In another mode, the shape deviation has been followed on the basis of point cloud data obtained by digitizing of an unknown surface.

In both cases, algorithm of minimal zone (MZ) fitting was applied in order to obtain an associative geometry. Type A standard uncertainty has been estimated according to five factor replications. Software Minitab was used to assess the 
impact of the aforementioned factors and their interactions on the standard uncertainty.

The analysis of standard deviation when the complex surface was regarded as a set of basic geometric primitives has shown that the standard deviation of the torus was much larger than the standard deviation of the spheres, therefore, we calculated measurement uncertainty for the measurement of the torus only. Fig.2. shows the effect of individual factors and their interactions on the standard deviation in the measurement of the torus whose majority of the area represents the complex surfaces. It is obvious from the figure that the most influential factor in the observed variable is scan direction. The highest standard deviation is obtained in interaction with the position of the workpiece on the machine table (in this case, both factors are on the "2" level). Analysis of variance (ANOVA) showed that at the level of significance $\alpha=0.05$ the relevant factors for standard deviation are as follows: the scanning direction, the position of the workpiece on the CMM table, their interactions and the interactions of scanning speed and density of sampling. Other factors and investigated interactions were not statistically relevant at this threshold of significance. Fig.3. shows the effect of individual factors and their interactions on the standard deviation in the measurement of complex surfaces as an unknown area. It is obvious that the highest standard deviation was obtained when the density of points was on the "1" level and the configuration of the measuring probe was on the "2" level.

In Figures 2 and 3 the $\mathrm{x}$ axis indicates the factor level while $y$ axis shows the value of the form medium error of repeated measurements expressed in $\mu \mathrm{m}$. According to an analysis of variance (ANOVA) these two factors were statistically significant $(\alpha=0.05)$, and the largest effect had sampling density. Other factors were not statistically relevant at this threshold of significance.

Calculated uncertainties for both measurement tasks are given in Table 3. [22]

Table 3. Calculated uncertainties for both measurement tasks.

\begin{tabular}{|c|c|c|c|c|c|}
\hline $\begin{array}{c}\text { Test } \\
\text { no. }\end{array}$ & $\begin{array}{c}\boldsymbol{U}_{\mathbf{F}} \\
{[\boldsymbol{\mu \mathbf { m } ]}}\end{array}$ & $\begin{array}{c}\boldsymbol{U}_{\mathbf{F F}} \\
{[\boldsymbol{\mu \mathbf { m } ]}]}\end{array}$ & $\begin{array}{c}\text { Test } \\
\mathbf{n o .}\end{array}$ & $\begin{array}{c}\boldsymbol{U}_{\mathbf{F}} \\
{[\boldsymbol{\mu \mathbf { m } ]}]}\end{array}$ & $\begin{array}{c}\boldsymbol{U}_{\mathbf{F F}} \\
{[\boldsymbol{\mu \mathbf { m } ]}]}\end{array}$ \\
\hline 1 & 2.39 & 2.91 & 9 & 2.97 & 0.99 \\
\hline 2 & 2.43 & 1.65 & 10 & 3.46 & 2.05 \\
\hline 3 & 2.47 & 6.47 & 11 & 2.48 & 2.23 \\
\hline 4 & 2.88 & 5.08 & 12 & 2.44 & 1.95 \\
\hline 5 & 2.41 & 0.65 & 13 & 3.19 & 2.09 \\
\hline 6 & 2.38 & 1.35 & 14 & 3.15 & 2.48 \\
\hline 7 & 2.78 & 0.96 & 15 & 2.48 & 2.29 \\
\hline 8 & 3.38 & 1.83 & 16 & 2.82 & 4.97 \\
\hline
\end{tabular}

In most cases Type B evaluation of standard uncertainty is based on scientific judgment using all relevant information of the measurement system. This may include the manufacturer's specification, historical data, calibration data, and general knowledge of the measurement system. Three probability distributions are used to transform the limits of the relevant information into a standard uncertainty, they are Gauss distribution, Rectangular, and U distribution. Usually, we take Rectangular distribution [23].

Starting from the specifications of the machine the maximum permissible error is $+/-(1.9+L / 330) \mu \mathrm{m}$ and it can be considered as a framework in which each measurement result should lie in, this statement is characterized by uncertainty $u_{\mathrm{B} 1}$.

In our case, the size of the torus $(62 \mu \mathrm{m})$ was not the calibrated measure. The temperature effect is taken into account through the difference between the coefficient of thermal expansion of CMM and measured part (aluminum) according to (7):

$$
C T E_{|C M M-P a r t|}=11.5-24=12.5 \frac{\mathrm{ppm}}{{ }^{\circ} \mathrm{C}},
$$

where CTE represents the difference between the coefficients of thermal expansion between the CMM and part to be measured.

Temperature uncertainty in areas where the measurement was carried out can be considered to be $+/-0.2^{\circ} \mathrm{C}$, so the uncertainty Type $\mathrm{B}$ will be $u_{\mathrm{B} 2}$. Three other standard uncertainties were derived from temperature effects. Two of them are due to uncertainties in the coefficients of thermal expansion of the CMM and working parts and are provided as a $10 \%$ uncertainty for the CTE value. The third one is related to measurement time. They are characterized by $u_{\mathrm{B} 3}$. $u_{\mathrm{B} 4 \text {. }}$

In most cases it is expected that the uncertainty in temperature during the measurements is lower compared to the temperature uncertainty in the room. This assumption is considered to be valid as the actual time of measurement is less than the period required for determining the uncertainty of the room temperature. Finally, the standard uncertainty that is used to calculate the combined uncertainty is the uncertainty of the calibration of standards $u_{\mathrm{B} 5}$. Total $u_{B}$ component is the square root of the sum of squares $u_{B i}$ as it is listed in Table 4.

Table 4. Calculated uncertainties Type B.

\begin{tabular}{|c|c|c|c|}
\hline $\begin{array}{l}\text { Contri- } \\
\text { bution }\end{array}$ & Calculation & $\begin{array}{l}\text { Distri- } \\
\text { bution }\end{array}$ & $\begin{array}{l}\text { Value } \\
{[\mu \mathrm{m}]}\end{array}$ \\
\hline$u_{\mathrm{B} 1}$ & $\frac{0.6+(1.5 \times 62 / 330)}{\sqrt{3}}$ & $\begin{array}{c}\text { Uniform } \\
\text { (rectangul } \\
\text { ar) }\end{array}$ & 0.372 \\
\hline$u_{\mathrm{B} 2}$ & $\frac{12.5 \times 62 \times 0.2}{\sqrt{3}}$ & Uniform & 0.0896 \\
\hline$u_{\mathrm{B} 3}$ & $\frac{0.015 \times 30.0005 \times 0.2}{\sqrt{3}}$ & Uniform & 0.00006 \\
\hline$u_{\mathrm{B} 4}$ & $\frac{11.35 \times 30.0005 \times 0.07}{\sqrt{3}}$ & Uniform & 0.0137 \\
\hline$u_{\mathrm{B} 5}$ & & $\begin{array}{c}\text { Constant } \\
\text { value }\end{array}$ & 0.000085 \\
\hline$u_{\mathrm{B}}$ & $\sqrt{u_{B 1}^{2}+u_{B 2}^{2}+u_{B 3}^{2}+u_{B 4}^{2}}$ & & 0.38505 \\
\hline
\end{tabular}



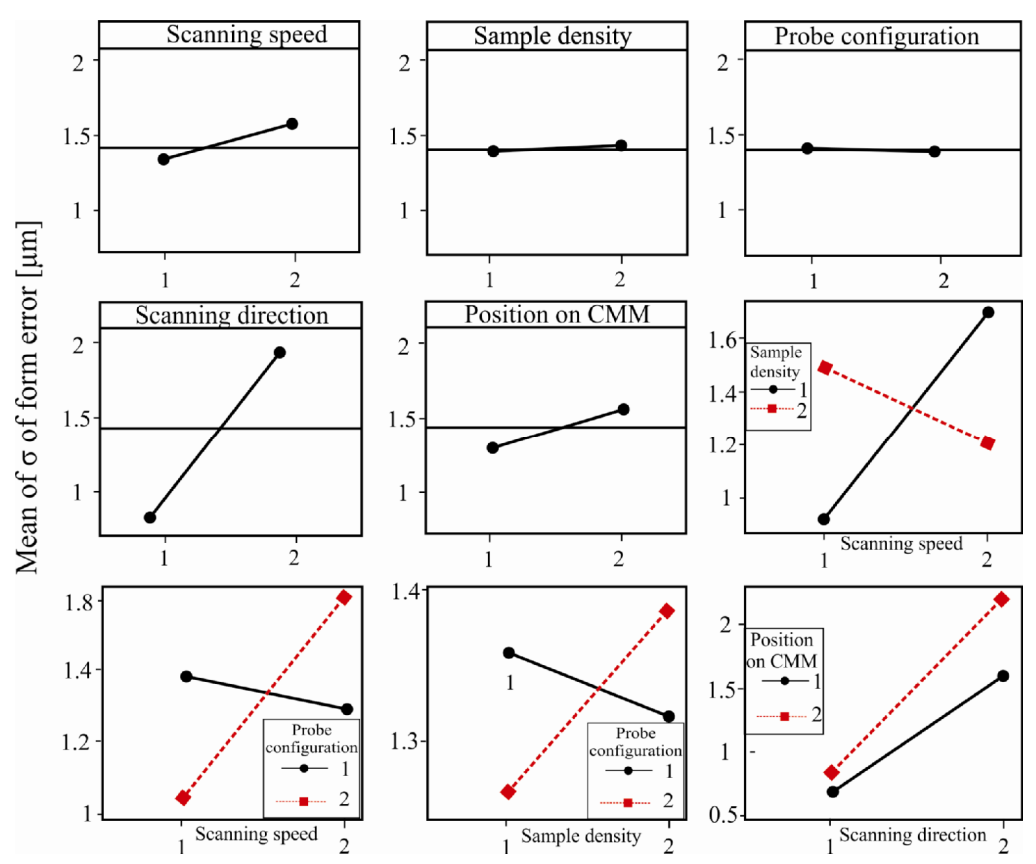

Fig.2. Effect of individual factors and their interactions on the standard deviation in the measurement of the torus.
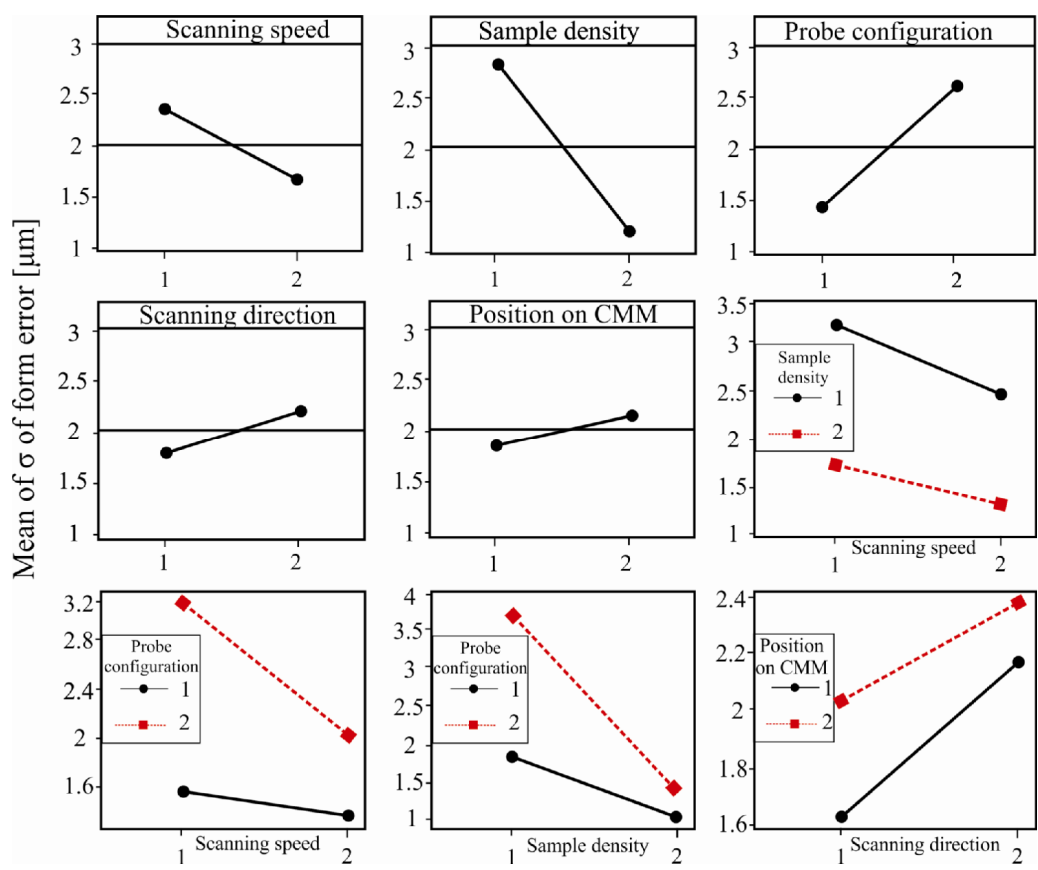

Fig.3. Main Effects Plot and Interaction Plot(data means) for $\sigma$ of form error $[\mu \mathrm{m}]$.

\section{DISCUSSION / CONCLUSIONS}

Our investigations were focused on the evaluation of task specific measurement uncertainty when using coordinate measuring machines. Although the focus of this work was for CMMs equipped with touch probes, its results can be used for CMMs equipped with other types of probing systems. The experimental work and literature review indicated that measurement strategy was a significant factor that influenced measurement uncertainty. Due to the number of variables present in a CMM system, the evaluation of task specific measurement uncertainty can be a very complex task. However, there are different approaches which can aid the estimation of measurement uncertainty as are sensitivity analysis, expert judgment, substitution, simulation, and measurement history. In essence, we have chosen a more traditional metrology approach, the sensitivity analysis approach known as uncertainty budgeting, consisting of listing each uncertainty source, its magnitude, effect on the measurement result, correlation with other uncertainty sources, and combining appropriately. 
Both methods used for uncertainty assessment showed good correlation, although the ISO 15530-3 method was found to be more sensitive to changes in the CMM repeatability when compared with GUM approach.

The temperature effects during CMM measurements were recognized as the major contributor to the measurement uncertainty. Temperature did not influence the repeatability of the measurement results and caution is required when applying temperature variations to an uncertainty model.

The Design of Experiments approach to uncertainty estimation is focused on understanding how the selected input factors of the CMM system affect the output response. Furthermore, the Design of Experiments approach also allows the experimenter to study the interactions between such factors depending on the type of DOE method selected for the study.

Future research will be directed towards the development and application of simulation methods. The simulation approach provides a more comprehensive access to the estimation of measurement uncertainty because all or most contributors can be described individually or described under certain assumptions. Such approach allows the user to determine how significantly each of the individual factors contributes towards the expanded uncertainty. It is important to recognize that the measurement uncertainty is task specific and as such there will be factors which remain constant in terms of their influence during the measurement process and factors that may vary from task to task.

\section{ACKNOWLEDGMENT}

The work was supported by the Slovak University of Technology, Faculty of Mechanical Engineering, and the Slovak Research and Development Agency, grant APVV0096-10 and by the research grants VEGA 1/0120/12 and VEGA 1/0604/15.

\section{REFERENCES}

[1] International Organization for Standardization. (1998). Geometrical Product Specifications (GPS) Inspection by measurement of workpieces and measuring equipment - Part 1: Decision rules for proving conformance or non-conformance with specifications. ISO 14253-1.

[2] Trapet, E., Savio, E., De Chiffre, L. (2004). New advances in traceability of CMMs for almost the entire range of industrial dimensional metrology needs. CIRP Annals - Manufacturing Technology, 53, 433-438.

[3] Acko, B., Mccarthy, M., Haertig, F., Buchmeister, B. (2012). Standards for testing freeform measurement capability of optical and tactile coordinate measuring machines. Measurement Science \& Technology, 23 (9), 094013.

[4] Wilhelma, R.G., Hockena, R., Schwenkeb, H. (2001). Task specific uncertainty in coordinate measurement. CIRP Annals - Manufacturing Technology, 50, 553-563.
[5] International Organization for Standardization. (2004). Geometrical Product Specifications (GPS) Coordinate measuring machines (CMM): Technique for determining the uncertainty of measurement - Part 3: Use of calibrated workpieces or standards. ISO/TS 15530-3.

[6] International Organization for Standardization. (2008). Geometrical Product Specifications (GPS) Coordinate measuring machines (CMM): Technique for determining the uncertainty of measurement - Part 4: Evaluating task-specific measurement uncertainty using simulation. ISO/TS 15530-4.

[7] Savio, E., De Chiffre, L. (2003). Validation of calibration procedures for freeform parts on CMMs. In International Topical Conference on Precision Engineering, Micro Technology, Measurement Techniques and Equipment (EUSPEN), 19-20 May 2003, Aachen, Germany, 539-542.

[8] Savio, E., De Chiffre, L. (2002). An artefact for traceable freeform measurements on coordinate measuring machines. Precision Engineering, 26, 58-68.

[9] Savio, E., Hansen, H.N., De Chiffre, L. (2002). Approaches to the calibration of freeform artefacts on coordinate measuring machines. CIRP Annals Manufacturing Technology, 51, 433-436.

[10] Barini, E.M., Tosello, G., De Chiffre, L. (2010). Uncertainty analysis of point-by-point sampling complex surfaces using touch probe CMMs: DOE for complex surfaces verification with CMM. Precision Engineering, 34, 16-19.

[11] Flack, D. (2011). Good Practice Guide No. 42 - CMM Verification. NPL Good Measurement Practice. Teddington, England: National Physical Laboratory.

[12] Acko, B., Sluban, B., Tasic, T., Brezovnik, S. (2014). Performance metrics for testing statistical calculations in interlaboratory comparisons. Advances in Production Engineering \& Management, 9 (1), 44-52.

[13] Crepinsek-Lipus, L., Matus, M., Acko, B. (2013), Optimization of calibrating HeNe laser interferometers by sample-period simulation. International Journal of Simulation Modeling, 12 (3), 154-163.

[14] Rodger, G., Flack, D., McCarthy, M. (2007). A Review of Industrial Capabilities to Measure Free-Form Surfaces. NPL Report DEPC - EM 014. Teddington, England: National Physical Laboratory.

[15] Feautrier, D., Bourdet, P. (1997). Form error assessment: Error separation method for the calibration of free-form surfaces by means of co-ordinate measuring machines. In Proceedings of the Annual Meeting-American Society for Precision Engineering. ASPE, 16, 82-85.

[16] Piratelli-Filho, A., Di Giacomo, B. (2003). CMM uncertainty analysis with factorial design. Precision Engineering, 27, 283-288.

[17] Feng, C.J., Saal, A.L, Salsbury, J.G., Ness, A.R., Lin, G.C.S. (2007) Design and analysis of experiments in CMM measurement uncertainty study. Precision Engineering, 31, 94-101. 
[18] Feng, C.X., Wang, X. (2002). Subset selection in predictive modeling of CMM digitization uncertainty. Journal of Manufacturing Systems, 21, 419-439.

[19] Sun, A., Anand, S., Tang, J. (2002). Comprehensive design of experiments based framework for optimal CMM inspection and uncertainty analysis of form tolerances. International Journal of Production Research, 40, 2097-2123.

[20] Peace, S.G. (1993). Taguchi Methods: A Hands-On Approach. Adison-Wesley.

[21] International Organization for Standardization. (1995). GUM - Guide to the expression of uncertainty in measurement.
[22] Vrba, I. (2013) Uncertainty estimation for coordinate measuring machines using for complef surfaces measurement. Unpublished doctorial dissertation. Slovak Technical University, Bratislava, Slovakia.

[23] Tasic, T., Acko, B. (2011). Integration of a laser interferometer and a CMM into a measurement system for measuring internal dimensions. Measurement, 44 (2), 426-433.

Received November 24, 2014. Accepted June 23, 2015. 